\title{
OTS: A program for converting Noldus Observer data files to SDIS files
}

\author{
ROGER BAKEMAN \\ Georgia State University, Atlanta, Georgia \\ and \\ VICENÇ QUERA \\ University of Barcelona, Barcelona, Spain
}

\begin{abstract}
A program for converting Noldus Observer data files (ODF) to sequential data interchange standard (SDIS) files is described. Observer users who convert their data files can then take advantage of various flexible and powerful data modification and computational procedures available in the Generalized Sequential Querier, a program that assumes SDIS-formatted files.
\end{abstract}

The Observer (Noldus, 1991, 1996; Noldus, Trienes, Hendriksen, Jansen, \& Jansen, 2000; for reviews, see Albonetti et al., 1992) is a sophisticated software package for the collection and analysis of observational data. Data may be collected initially using handheld devices and then uploaded to a personal computer (PC) later, may be entered directly into the PC either live or from any of a variety of records, or coded from videotapes or digital media files. In any event, Observer data files (ODFs) result.

The sequential data interchange standard (SDIS) is a standard format for sequential data (Bakeman \& Quera, 1992), and the Generalized Sequential Querier (GSEQ) is a program that analyzes SDIS-formatted data (Bakeman \& Quera, 1995). Both The Observer and GSEQ, when first introduced, ran on IBM-compatible PCs, first under DOS and later as a DOS window under Windows. Windows versions of both program are now available. The Observer is commercially available from Noldus Information Technology, and GSEQ for Windows (GSW) can be downloaded from the authors' web pages (see Availability section) into a directory that contains the earlier version of GSEQ included with Bakeman and Quera (1995).

Here we describe a computer program that converts ODF- to SDIS-formatted data files. The OTS program is useful for investigators who use The Observer to collect data but who then wish to take advantage of the extensive data modification and analytic capabilities of GSEQ (Bakeman \& Quera, 1995). The more powerful modifications are time based. For example, users can define new codes that occur only at those times when two or more earlier behaviors were all coded (a logical and), or at times when any one of a set of behaviors was coded (a logical or). Other logical operations are also available. In ad-

Correspondence should be addressed to R. Bakeman, Department of Psychology, Georgia State University, Atlanta, GA 30303 (e-mail: bakeman@gsu.edu). dition, users can define new codes that are tied to onset and offset of existing codes. For example, a new code might include the $10 \mathrm{sec}$ before and the $5 \mathrm{sec}$ after the onset of an alarm cry. GSW allows for lag sequential analysis to any of a number of positive or negative lags and also includes a program that converts time- to event-based data. Another useful feature, especially when observers are first being trained, is a program that lets the user define codes within a specified time window as an agreement and then computes the $\kappa$ agreement statistic for 2 observers using this flexible window.

\section{Sampling Method}

The Observer recognizes four sampling methods: focal sampling (continuous recording), ad-lib sampling, instantaneous sampling, and one-zero sampling. The method is specified initially for a given research project when one is creating the Observer configuration file, and it applies to all ODFs created for that project. The version of OTS described here (Version 1.1) only converts ODFs collected using focal sampling; throughout this paper, focal sampling is assumed.

\section{ODFs and SDIS Analytic Units}

Files and units. Almost always, investigators bundle sequences of observational data into units of some sort for subsequent analysis. The Observer creates a separate ODF for each of these bundles and then keeps a list of ODF names associated with each project in a project file. SDIS (Bakeman \& Quera, 1995), on the other hand, terms these bundles subjects, and places data from all subjects associated with a study in a single data file.

The term subject is traditional but limited. We now prefer the term analytic unit or simply unit, partly because it recognizes that the unit need not be an individual but may be a dyad or group, or the same individual observed at different ages. Statistics and indices derived from observational data for a unit, however unit is defined, are often 
analyzed later using conventional statistical techniques such as multiple regression or log-linear analysis. For example, different mother-infant pairs might be observed, and thus the pair would serve as the unit of analysis. Applying this terminology, each ODF contains data for an analytic unit.

Each ODF is identified with a title that the user enters when starting data collection, the date and time, and values for independent variables (information that characterizes the bundle). SDIS identifies each unit with a name, which is limited to 16 characters and is enclosed in angle brackets (e.g., <\#1>). The OTS program uses the first 16 characters of the ODF title as the unit name. If no title was entered, then OTS substitutes \#n instead, where $n$ is a sequential number.

Observations and sessions. The Observer creates an ODF for each data collection session. The Base Package for Windows Reference Manual Version 3.0 (Noldus, 1996) defines the "observational data entered into the computer between time $=0$ (start of observation) and time $=\{$ end $\}$ (end of observation)" as an observation (p. A-19). SDIS (Bakeman \& Quera, 1995) defines a "sequence of coded events for which continuity can be assumed" as a session (p. 22). Thus an observation to The Observer 3.0 (and Version 4.0 as well) is a session to SDIS.

Whatever terms are used, a pair is needed. The subordinate term indicates an uninterrupted recording and the superordinate term a collection of such recordings that share common descriptors. The Observer 3.0 (and 4.0) uses observation for the subordinate term and does not use the superordinate term. Bakeman and Quera (1995) used session for the subordinate term and subject for the superordinate term. The Observer 5.0 plans to use observation for the subordinate term and session for the superordinate term (A. Hendriksen, personal communication, January 19, 1999; L. P. J. J. Noldus, personal communication September 3, 1999). Here, in the interests of both clarity and consistency, we use recording for the subordinate term (instead of session) and continue to use unit for the superordinate term, thereby avoiding the potentially confusing session.

SDIS permits a unit to consist of one or more recordings, separating each with a semicolon; thus the general form of a unit in an SDIS file is

\section{$<$ identifier $>$ uninterrupted recording [; uninterrupted recording]/}

where square brackets enclose optional material and the slash indicates the end of data for that unit. Any breaks in continuity might be planned (e.g., one recording per day for a week for one child) or unplanned (e.g., an unexpected event intrudes during data collection), and are different from the suspend and resume recording, which The Observer allows and which are discussed later.

In similar fashion, Version 5.0 but not Version 3.0 or 4.0 of The Observer permits more than one recording to appear in an ODF, in which case the general form of an ODF is

identifying_information
$\ldots$.
$\{$ start $\}$
$\ldots$
time $\{$ end $\}$
$[\{$ start $\}$
$\ldots$
time $\{$ end $\}]$

When an ODF contains more than one recording, OTS separates them with a semicolon.

\section{Observer and SDIS Variables}

The Observer permits users to define up to 40 independent variables, which describe aspects of a data collection or recording session that do not change, such as the animal or person observed, its sex, or the name of the observer. Independent variables may be useful descriptively or they may define factors that reflect the design of the study.

SDIS permits users to define up to seven variables, corresponding to factors that reflect the design of the study, along with up to 12 levels (i.e., conditions) for each variable. For example, a factor might be sex with two levels (male, female) or treatment with two conditions (experimental, control). Variables are useful primarily when values for each level are exported to standard statistical programs along with summary statistics for each analytic unit (i.e., each ODF). Additionally, all summary tables produced by GSEQ may, optionally, be pooled over conditions.

The Observer permits two types of independent variables, character and numeric. If character, then all levels (i.e., options) may be specified (Free Format not checked) or only some or none may be specified (Free Format checked). Only character independent variables without Free Format checked are eligible to be selected as SDIS variables. OTS asks the user to identify which of these ODF independent variables are to serve as SDIS variables and which are to be included in the SDIS-formatted file as information only. Values of variables that are indicated as information only are included in SDS files as comments (we often call an SDIS-formatted file an SDS file because the extension for such files is SDS).

\section{Observer and SDIS Data File Elements}

Subjects. The Observer permits users to specify either single or multiple actors. If single, each observation session is of a single actor, so no actor codes need appear in the ODF. If multiple, codes for actors are included in the data files. Before collecting data, the Observer user defines a 1-3 character code for up to 120 actors (i.e., subjects) along with a longer name (up to 8 characters) and an even longer definition, if desired.

Behavioral elements and modifiers. In the simplest instance, Observer users enter a behavior (symbolized here as $B e h$ ). The program then supplies the time. Behaviors may be preceded by actors (symbolized here as $A c t$ ), if multiple, and may be followed by one or two modifiers 
(symbolized here as Mod). Comments may also be included. Thus, a typical data line in an ODF is

$$
\text { time [Act,] Beh [,Mod] [,Mod] [,/* comment], }
$$

where square brackets indicate an optional element.

Behavioral elements and modifiers, like actors, are represented with a $\mathbf{1}-\mathbf{3}$ character code as well as a longer name (up to 8 characters) and an even longer, more descriptive definition. The first and second modifiers can be an actor or a behavior or one of the modifiers explicitly declared by the user. Before collecting data, Observer users specify whether ODFs are to contain the briefer codes or the longer names for the various elements (actor, behavioral, modifier).

Behavioral element and modifier classes. Behavioral elements and modifiers are grouped into classes, each of which is identified with a unique name. In addition, behavioral elements are classified as either state or event. In Observer terminology, states represent duration behaviors and events represent momentary behaviors (cf. Bakeman \& Gottman, 1997). The durations of momentary behaviors are not noted; only their onset times are recorded by The Observer. Onset times for duration behaviors are likewise recorded, but they are assumed to continue until the next behavior in the same class is recorded, which signals the offset of the previous behavior in the class. Thus the state codes in each behavioral class are assumed to be mutually exclusive and exhaustive. Likewise, codes in each modifier class are assumed to be mutually exclusive and exhaustive.

Before collecting data, Observer users specify which modifiers are permitted for each behavioral element. For the first modifier, the user might specify none, an actor, a behavioral element, or any modifier from a specified class. If no first modifier was specified, a second modifier cannot be given. However, if a first modifier was specified, the second can likewise be specified as none, an actor, a behavioral element, or a modifier from a specified class. Thus, when qualifying a behavioral element, and assuming the modifier is not an actor or a behavioral element, the first modifier may come from only one modifier class; likewise for the second.

Thus, possible lines from an ODF include

$$
\begin{aligned}
& \text { time [Act,] Beh } \\
& \text { time [Act,] Beh, } \mathrm{MC}_{i} \\
& \text { time [Act,] Beh, } \mathrm{MC}_{i}, \mathrm{MC}_{j} \text {, } \\
& \text { time [Act,] Beh, Act, } \mathrm{Beh} \\
& \text { time [Act,] Beh, Act, } \mathrm{MC}_{i}
\end{aligned}
$$

where $\mathrm{MC}_{i}$ represents a modifier from the $i$ th modifier class. Any of these can end with a comment.

SDIS codes. SDIS does not divide codes into actors, behavioral elements, and modifiers, as The Observer does, but instead regards all codes as alike. Thus, whereas The Observer provides a structured input consisting of up to four elements (Act, Beh, Mod, Mod), SDIS is more free form. This provides both options and flexibility for converting ODFs into SDIS-formatted files.

\section{Observer to SDIS Conversion Examples: Single Actor}

For all examples in this section, assume that number of actors was specified as single when the Observer configuration file was being created.

One behavioral class. Imagine for example that Vocal is a class of behavioral elements containing states Talk, Cry, and Quiet and that no other behavioral classes, actors, or modifiers are defined. For this and later examples, assume that codes consist of the first two letters of each name and that the Observer's data file format was set to normal and not compressed (i.e., the ODF contains the longer names and not the briefer codes to represent the various elements). Then, OTS, which always uses The Observer's codes and not names, would convert as follows:

$$
\begin{array}{cl}
\begin{array}{c}
\text { Observer Data File } \\
\text { start\} }
\end{array} & \multicolumn{1}{c}{\text { SDS Data File }} \\
\text { 0 Quiet } & <\# 1> \\
5 \text { Talk } & , 0 \mathrm{Qu}, 0-\mathrm{Ta}, 5-\mathrm{Cr}, 12-\mathrm{Qu}, 18-, 29 / \\
\text { 12 Cry } & \\
18 \text { Quiet } & \\
29 \text { end } &
\end{array}
$$

SDIS includes five data types and, within each data type, several formatting options. When converting focal sampling (continuous recording) ODFs, the OTS program uses some of the options available to what SDIS terms timed-event sequential data (TSD). This data type is declared at the outset of the SDS file. It is followed by the codes in parentheses, indicating that these codes form a mutually exclusive and exhaustive set. The declaration is followed by a unit name, the onset time for the session, the onset times for codes, and the offset time. A semicolon terminates the declaration and a slash terminates each unit.

The declaration of codes after the type is optional and may be omitted by checking the Omit explicit code declaration; let SDIS order codes option in OTS. Declaring codes explicitly is useful when SDS files are created by data entry because then any undeclared codes found in the file are flagged as errors. However, when converting ODFs, this is not an issue. Still, OTS users may prefer explicit declaration because then GSEQ will output results for codes following the order in which they are declared instead of the order in which they first appear in the data file.

Two behavioral classes. As a second example, imagine that Posture is a second class of behavioral elements containing states Sit, Lie, and Stand and an event (momentary) code Wave and that the Vocal class included an event code Burp. Then OTS would convert as follows:

\begin{tabular}{cl}
$\begin{array}{c}\text { Observer Data File } \\
\text { \{start }\end{array}$ & \multicolumn{1}{c}{ SDS Data File } \\
0 Quiet & $<\# 1>$ \\
0 Lie & $, 0 \mathrm{Qu}, 0-\mathrm{Ta}, 5-\mathrm{Cr}, 12-\mathrm{Qu}, 18-\&$ \\
4 Sit & $\mathrm{Li}, 0-\mathrm{Si}, 4-\mathrm{Li}, 12-\mathrm{Si}, 24-\mathrm{St}, 27-\&$ \\
5 Talk & $\mathrm{Bu}, 7 \mathrm{Bu}, 10 \mathrm{Wa}, 14,29 /$
\end{tabular}



7 Burp
10 Burp
12 Cry
12 Lie
14 Wave
18 Quiet
24 Sit
27 Stand
29 \{end

When converting focal sampled ODFs, OTS places each class of behavioral elements in separate streams, reserving a final stream for any momentary (event) codes. As is evident from the example, streams in SDIS are separated with ampersands; the onset time for the session appears at the beginning of the first stream and the offset time for the session appears at the end of the last stream, just before the terminating slash. Hyphens after a time indicate a duration (state) code that continues until the next code in the class begins (or until the session ends), whereas the absence of a hyphen after a time indicates a momentary (event) code that lasts, in effect, one time unit.

Two behavioral classes with modifiers. As a third example, imagine that three actors are defined named Andy, Barb, and Carl; that Strength is a class containing modifiers Low and High; that Clarity is a class containing modifiers Clear and Fuzzy; and that behavioral elements Talk and Wave are modified by an Actor, behavioral element Cry is modified by Strength and Clarity, behavioral element Burp by Strength, and behavioral element Sit by Clarity. Then OTS would convert as follows:

\begin{tabular}{|c|c|}
\hline $\begin{array}{l}\text { Observer Data File } \\
\text { \{start }\}\end{array}$ & $\begin{array}{l}\text { SDS Data File } \\
\text { Timed BuLo BuHi WaAn WaBa } \\
\text { WaCa }\end{array}$ \\
\hline 0 Quiet & (TaAn TaBa TaCa CrLoCl CrLoFu \\
\hline 0 Lie & $\begin{array}{l}\mathrm{CrHiCl} \mathrm{CrHiFu} \mathrm{Qu})(\mathrm{SiCl} \mathrm{SiFu} \mathrm{Li} \\
\quad \mathrm{St})\end{array}$ \\
\hline 4 Sit,Fuzzy & $<\# 1>$ \\
\hline 5 Talk,Carl, & $\begin{array}{l}\text {,0 Qu,0- TaCa,5-CrLoFu,12- } \\
\text { Qu,18- \& }\end{array}$ \\
\hline 7 Burp,High & $\begin{array}{l}\mathrm{Li}, 0-\mathrm{SiFu}, 4-\mathrm{Li}, 12-\mathrm{SiCl}, 24- \\
\quad \mathrm{St}, 27-\&\end{array}$ \\
\hline $\begin{array}{l}10 \text { Burp,Low } \\
12 \text { Cry,Low,Fuzzy } \\
12 \text { Lie } \\
14 \text { Wave,Andy } \\
18 \text { Quiet } \\
24 \text { Sit,Clear } \\
27 \text { Stand } \\
29 \text { end }\end{array}$ & BuHi,7 BuLo, 10 WaAn, $14,29 /$ \\
\hline
\end{tabular}

By default, OTS creates combination codes representing behavioral elements with their modifiers (e.g., CrLoFu for Cry,Low,Fuzzy).

Investigators may wish to analyze behavioral elements as separate codes so that, for example, the proportion of time spent crying, no matter how modified, could be de- termined. In that case users should check the Create separate behavior and modifier codes option. If they do, OTS would convert the file just given as follows:

\section{SDS Data File}

Timed Bu Wa (Ta Cr Qu) (Si Li St) (VoAn VoBa VoCa) (PoAn PoBa PoCa) (Lo Hi) ( $\mathrm{VoCl} \mathrm{VoFu)} \mathrm{(PoCl} \mathrm{PoFu);}$ $<\# 1>$

,0 Qu,0- Ta+VoCa,5- Cr+Lo+VoFu, 12- Qu, 18- \& $\mathrm{Li}, 0-\mathrm{Si}+\mathrm{PoFu}, 4-\mathrm{Li}, 12-\mathrm{Si}+\mathrm{PoCl}, 24-\mathrm{St}, 27-\&$

$\mathrm{Bu}+\mathrm{Hi}, 7 \mathrm{Bu}+\mathrm{Lo}, 10 \mathrm{Wa}+\mathrm{PoAn}, 14$,29/

The notation $\mathrm{Ta}+\mathrm{VoCa}$ indicates that codes $\mathrm{Ta}$ (talk) and $\mathrm{VoCa}$ (Carl is the recipient of a behavior in the vocal class) both begin at Time 5 and continue to Time 12. You may wonder why $\mathrm{VoCa}$ and not $\mathrm{Ca}$. When all behavioral elements that specify actor or a modifier class (in essence, actor is a special modifier class) themselves belong to the same class (as is often the case), then the separate codes produced by OTS would be the same as the codes specified in The Observer (e.g., the modifier class Strength modified only codes in the vocal class, so $H i$ and $L o$ appear in the converted data). However, when behavioral elements from more than one class specify actor or the same modifier class, then the modifier is prefixed with a code indicating the class (e.g., $V o F u$, indicating that Fuzzy modified a vocal-class code, and $P o F u$, indicating that Fuzzy modified a posture-class code).

Without the class prefixes (e.g, here $V o$ and $P o$ ), there would be no way to know which class of behavior was being modified; moreover, two $\mathrm{Fu}$ codes could occur at the same time, for example, which the SDIS compiler would flag as an error. The Observer asks users to provide class names but not class codes. OTS, however, may need class codes to use as prefixes, and if so prompts the user to supply them.

You may wonder whether or not you should check the Create separate behavior and modifier codes option. In part it depends on the sorts of analyses you plan, but in one sense it does not matter because, no matter which option is selected, combination or separate codes can be created later using GSEQ data modification commands. For example, if you had not checked the Create separate behavior and modifier codes option, then the command

$$
\text { OR Talk = TaAn TaBa TaCa; }
$$

would create a new code, Talk, for any time unit (e.g., each second if a 1-sec timing resolution was specified initially) already coded for $T a A n, T a B a$, or $T a C a$. Similarly, if you wanted to know how often an actor was the recipient of a behavior, no matter the behavior, then

$$
\text { OR ToAndy = TaAn WaAn; }
$$

would produce a new code, ToAndy, for any time unit coded for either TaAn or WaAn. However, if you had checked the Create separate behavior and modifier codes option, then 
AND $\mathrm{TaCa}=\mathrm{Ta} \mathrm{VoCa} ;$

would produce a new code, $\mathrm{TaCa}$, for any time unit coded for both $\mathrm{Ta}$ and $\mathrm{VOCa}$, exactly like the code that would have resulted had you not checked the option.

You may prefer more mnemonic codes, for example, TalkCarl instead of TaCa. OTS creates combination codes from the 1-3 character Observer codes so as not to exceed the 16-character maximum set for SDIS codes. However, these combination codes can be changed if you wish. For example, the declaration

$$
\text { Timed TaCa CrLoCl CrLoFu ... }
$$

produced by OTS could be edited before being compiled by SDIS, using any text editor, to

$$
\begin{aligned}
& \text { Timed TalkCarl }=\text { TaCa CryLoClar }=\text { CrLoCl } \\
& \text { CryLoFuzzy }=\text { CrLoFu } . .
\end{aligned}
$$

The first name in the pair, before the equals sign, is the code as it appears in the SDS data file, but the second, after the equals sign, is used by GSEQ to label output. Alternatively, the GSEQ command

$$
\begin{aligned}
& \text { RENAME TalkCarl }=\mathrm{TaCa} \text { CryLoClear }=\mathrm{CrLoCl} \\
& \text { CryLoFuzzy }=\mathrm{CrLoFu} ;
\end{aligned}
$$

would likewise change $\mathrm{TaCa}, \mathrm{CrLoCl}$, and $\mathrm{CrLoFu}$ to more mnemonic names.

It should be noted that SDIS codes can be any combination of letters and digits, up to 16 characters, and may include the special characters $\#^{\wedge} \_[\text {] } \text { ? if the user wishes. }$

Four final comments: First, this example may seem somewhat complex but was deliberately chosen to show solutions to the problems that occur when behavioral elements from more than one class specify modifiers from the same class, even though we think this circumstance is not especially common.

Second, when the Create separate behavior and modifier codes option is checked, you may also want to check the Omit explicit code declaration option because the list of codes declared may become quite long and may include some codes that are never used.

Third, given a particular set of circumstances, it is possible for the SDIS compiler to find an error in an OTSproduced file. This would occur if the Create separate behavior and modifier codes option was checked, if both a state and an event behavioral element in the same behavioral class specified the same modifier class, and if the event was coded during time coded for the state with the same modifier code for both (e.g., ... Cr+Lo+VoFu, $12-\ldots \& \ldots \mathrm{Bu}+\mathrm{Hi}, 14 \ldots)$. SDIS would object to what it viewed as an overlapped code; the solution is to enclose the redundant event modifier code and time in percent signs (e.g., ... \% Bu+Hi, 14\% ...), which changes them into a comment.

Fourth, any comments in the data, which are signaled with a $/ *$ in the ODF, are included as a comment line in the SDS file.

\section{Observer to SDIS Conversion Examples: Multiple Actors}

For all examples in this section, assume that number of actors was specified as multiple when the Observer configuration file was being created. As a fourth example, again assume the same three actors as earlier and the same two behavioral classes, Vocal and Posture, but that for simplicity no modifiers and no momentary codes are specified. Because multiple actors are specified, an actor will precede each behavioral element. Then OTS would convert as follows:

\begin{tabular}{ll}
$\begin{array}{l}\text { Observer Data File } \\
\text { \{start\} }\end{array}$ & \multicolumn{1}{c}{ SDS Data File } \\
0 Andy,Quiet & <\#1> \\
0 Barb,Quiet & $, 0 \mathrm{AnQu}, 0-12 \mathrm{BaQu}, 0-5 \mathrm{CaQu}, 0-7$ \\
0 Carl,Quiet & BaTa,5-29 CaCr,7-18 AnCr,12-29 \\
0 Andy,Lie & CaQu,18-29 \& \\
0 Barb,Sit & AnLi,0-4 BaSi,0-12 CaLi,0-29 \\
& AnSi,4-27 \\
0 Carl,Lie & BaLi,12-24 BaSi,24-29 AnSt,27- \\
&, $29 /$ \\
4 Andy,Sit & \\
5 Barb,Talk & \\
7 Carl,Cry & \\
12 Andy,Cry & \\
12 Barb,Lie & \\
18 Carl,Quiet & \\
24 Barb,Sit & \\
27 Andy,Stand & \\
29 \{end &
\end{tabular}

When actors are multiple, OTS automatically checks the Omit explicit code declaration option because the number of possible codes can become quite large. Recognizing this problem, The Observer requires users to limit the number of actor-behavioral class combinations (called channels) to 32 for analysis. The example just given includes six such channels ( 3 actors $\times 2$ behavioral classes).

OTS uses somewhat different SDIS conventions for single and multiple actors. With single actors, OTS creates a stream for each behavioral class and an additional stream for any momentary codes. The onset times for codes within each stream are followed by a hyphen and a blank, indicating that the offset time for that code is the onset time of the next code in the stream. With multiple actors, explicit offset times are provided after the hyphen because the next code in a stream may involve a different actor, and that actor's onset time is not the offset time for the previous actor.

With multiple actors, users may also specify the Create separate behavior and modifier codes option. The example just given would be unchanged because there are no modifiers.

\section{Observer Control Codes}

ODFs include control codes, which are enclosed in curly brackets. For example, \{indvar\} signals that independent 
variable values follow, \{start $\}$ that data follows, \{end that data end, and \{note that notes entered after data collection follow. Other control codes can occur within the data. The following paragraphs detail these codes, their meanings, and how OTS interprets them.

\{mark\}. $\{$ mark $\}$ is inserted in the data when an Observer user selects the Insert Marker function. If the $A d d$ note to marker option was selected, a note can also be added. OTS treats the entire line as a comment.

\section{Observer Data File \\ 36.8 \{mark\} \\ Plane flying over}

\{susp\} and \{resu\}. \{susp $\}$ indicates a suspended observation, whereas \{resu signals that it has resumed. As noted earlier, SDIS permits a unit to consist of one or more separate sessions. Such sessions are often used to indicate a break in otherwise continuous recording, thus, \{susp $\}$ and $\{$ resu $\}$ in an ODF could be interpreted as an SDIS session break within a unit.

However, this would be incompatible with the way The Observer interprets \{susp\} and $\{$ resu\}. As noted in the Base Package for Windows Reference Manual, Version 3.0 (Noldus, 1996), "Behavioral states that are interrupted by a suspended period ... are treated as a single bout. The data analysis ignores the period between the \{susp $\}$ and $\{$ resu $\}$ codes" (pp. 6-60). Thus OTS simply notes the time taken by the break and subtracts that time from future codes, so that analysis results will be compatible with those produced by The Observer. However, the \{susp \} and \{resu\} are also noted with a comment in the SDS file so that a user could edit the SDS file later, adding semicolons where appropriate to indicate separate SDIS sessions.

\begin{tabular}{cl} 
Observer Data File & \multicolumn{1}{c}{ SDS Data File } \\
$\ldots$.15 A,Com & $\ldots$ \\
23 B,Agr & A+Com,15 B+Agr,23 C+Dis,32 \\
32 C,Dis & $\% 38$ susp $\}$ \\
38 susp & $\% 44$ resu \\
44 resu\} & 42 B+Com ... \\
48 B,Com ... &
\end{tabular}

This example assumes that the Create separate behavior and modifier codes option was checked.

\section{Converting Several ODFs to a Single SDIS File}

With The Observer, particular ODFs or parts of them are selected (i.e., filtered) for analysis, but the ODFs remain separate files. With SDIS, the units are incorporated in a single file, which is then subjected to analysis.
When converting ODFs into SDIS format, the user first selects an Observer project file (PRJ). The program displays a list of all ODFs associated with the project. Then the user selects which of those are to be converted.

Two options are possible. First, the user may request that all SDIS-formatted units be placed into a single SDS file. When placing all converted ODFs into a single SDS file, each ODF must reference the same configuration (CNF) file. This option is selected when the user plans to use GSEQ to analyze data from a project and so wants all units in a single file.

Alternatively, a user may request that each ODF be converted into a separate file. This can be useful when, for example, two ODF files represent two versions of the same events coded by different coders, and the user plans to use GSW's ComputeKappa program for continuous data in order to assess time-based observer agreement.

\section{Availability}

A zip file containing the OTS program may be down-

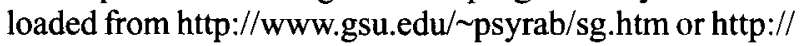
www.ub.es/comporta/sg.htm. The program assumes a Windows 95 or later environment.

\section{REFERENCES}

Albonetti, M. E., Lazarus, J., Dickins, D. W., Whybrow, A., Schönheiter, R., Prenter, J., Elwood, R., Newman, J. D., ÖDberg, F. O., Kaiser, L., Pham-Delègue, M. H., Verguelen, V., Fentress, J. C., \& Boccia, M. L. (1992). Software multiple review. The Observer: Software for behavioural research, Version 2.0. Ethology, Ecology, \& Evolution, 4, 401-414.

Bakeman, R., \& GOTTMAN, J. M. (1997). Observing interaction: An introduction to sequential analysis (2nd ed.). New York: Cambridge University Press.

BAKEMAN, R., \& QUERA, V. (1992). SDIS: A sequential data interchange standard. Behavior Research Methods, Instruments, \& Computers, 24, 554-559.

BaKeman, R., \& QueRa, V. (1995). Analyzing interaction: Sequential analysis with SDIS and GSEQ. New York: Cambridge University Press.

Noldus, L. P. J. J. (1991). The Observer: A software system for collection and analysis of observational data. Behavior Research Methods, Instruments, \& Computers, 23, 415-429.

Noldus, L. P. J. J. (1996). The Observer, base package for Windows. Reference manual, Version 3.0. Wageningen, The Netherlands: Noldus Information Technology.

Noldus, L. P. J. J., Trienes, R. J. H., Hendriksen, A. H. M., JANSEN, H., \& IANSEN, R. G. (2000). The Observer Video-Pro: New software for the collection, management, and presentation of timestructured data from videotapes and digital media files. Behavior Research Methods, Instruments, \& Computers, 32, 197-206.

(Manuscript received April 13, 1999; revision accepted for publication October 7,1999 .) 